\title{
Counterdiabatic control of transport in a synthetic tight-binding lattice
}

\author{
Eric J. Meier $\odot,{ }^{1}$ Kinfung Ngan, ${ }^{2}$ Dries Sels $, 3,4,5,{ }^{*}$ and Bryce Gadway $\oplus^{1, \dagger}$ \\ ${ }^{1}$ Department of Physics, The University of Illinois at Urbana-Champaign, Urbana, Illinois 61801-3080, USA \\ ${ }^{2}$ Department of Physics, The Chinese University of Hong Kong, Shatin, Hong Kong, China \\ ${ }^{3}$ Department of Physics, New York University, New York, New York 10003, USA \\ ${ }^{4}$ Center for Computational Quantum Physics, Flatiron Institute, New York, New York 10010, USA \\ ${ }^{5}$ Department of Physics, Harvard University, Cambridge, Massachusetts 02138, USA
}

(Received 2 June 2020; accepted 12 October 2020; published 9 November 2020)

\begin{abstract}
Quantum state transformations that are robust to experimental imperfections are important for applications in quantum information science and quantum sensing. Counterdiabatic (CD) approaches, which use knowledge of the underlying system Hamiltonian to actively correct for diabatic effects, are powerful tools for achieving simultaneously fast and stable state transformations. Protocols for CD driving have thus far been limited in their experimental implementation to discrete systems with just two or three levels, as well as bulk systems with scaling symmetries. Here, we extend the tool of CD control to a discrete synthetic lattice system composed of as many as nine sites. Although this system has a vanishing gap and thus no adiabatic support in the thermodynamic limit, we show that $\mathrm{CD}$ approaches can still give a substantial improvement in fidelity over naive, fast protocols.
\end{abstract}

DOI: 10.1103/PhysRevResearch.2.043201

\section{INTRODUCTION}

In the adiabatic limit, high-fidelity transport and statepreparation can be achieved by slowly deforming a system's Hamiltonian, such that population remains in a given instantaneous eigenstate of the system, whose properties may be tuned through the Hamiltonian. If the Hamiltonian is changed too quickly, however, the state will be unable to track the instantaneous eigenstate, resulting in poor transport or unfaithful state preparation. The cause for this breakdown in adiabaticity is that when the Hamiltonian begins to change in time, it effectively acquires additional terms that can couple the different instantaneous eigenstates. These additional diabatic terms are analogous to the inertial forces that appear for systems represented in accelerating reference frames, such as when considering the motion of an egg relative to a supporting spoon as a child sets off on a spirited race.

In both the classical and quantum contexts, a range of optimization protocols can be developed that allow one to surpass the adiabatic limit [1-10]. When there exists a known Hamiltonian for the system being deformed, the diabatic terms that would induce transitions between instantaneous eigenstates can, in principle, be precisely determined and, with sufficient resources, directly counteracted by appropriate measures. Approaches along such lines, known as counterdiabatic (CD)

\footnotetext{
*dsels@nyu.edu

†bgadway@illinois.edu

Published by the American Physical Society under the terms of the Creative Commons Attribution 4.0 International license. Further distribution of this work must maintain attribution to the author(s) and the published article's title, journal citation, and DOI.
}

techniques or shortcuts to adiabaticity, have been investigated in a physical context since the mid 2000s [1,11], beginning with works by Demirplak et al. [12,13] and Berry [14]. Theoretical formulations have been developed for small (twoand three-level) quantized systems $[15,16]$, as well as for transformations in continuous systems that have scaling transformations [17-19]. For the paradigmatic two-state problem, there have been demonstrations of counterdiabatic protocols across a wide range of experimental platforms, including atomic momentum states [20], nitrogen vacancy (NV) centers [21], and superconducting qubits [22-24]. More recently, CD protocols have even been demonstrated for the optimization of state transfer in three-level NV centers [25] and superconducting qutrits [26]. The extension to larger discrete quantum systems has been significantly challenged due to the general difficulty in both calculating the proper counterdiabatic terms to be used [27] and finding an experimental platform with the necessary level of control.

Here, we extend the application of $\mathrm{CD}$ techniques to discrete many-level quantum systems. Using synthetic tightbinding lattices of laser-coupled atomic momentum states, we implement counterdiabatic driving protocols [27] in a many-site system that has no adiabatic support in the thermodynamic limit. We demonstrate that $\mathrm{CD}$ driving protocols can lead to substantial improvements in state transformations across the lattice, such as in a multilevel adiabatic rapid passage as well as in the ability to prepare atoms in specific eigenstates delocalized across the synthetic lattices. The application of CD techniques in this context could lead to direct improvements in state transformations relevant to atom interferometry. Moreover, the native interactions in momentum-space lattices may enable future explorations into the influence of interactions on analog monopoles $[28,29]$, based on the connections between the physics of CD 


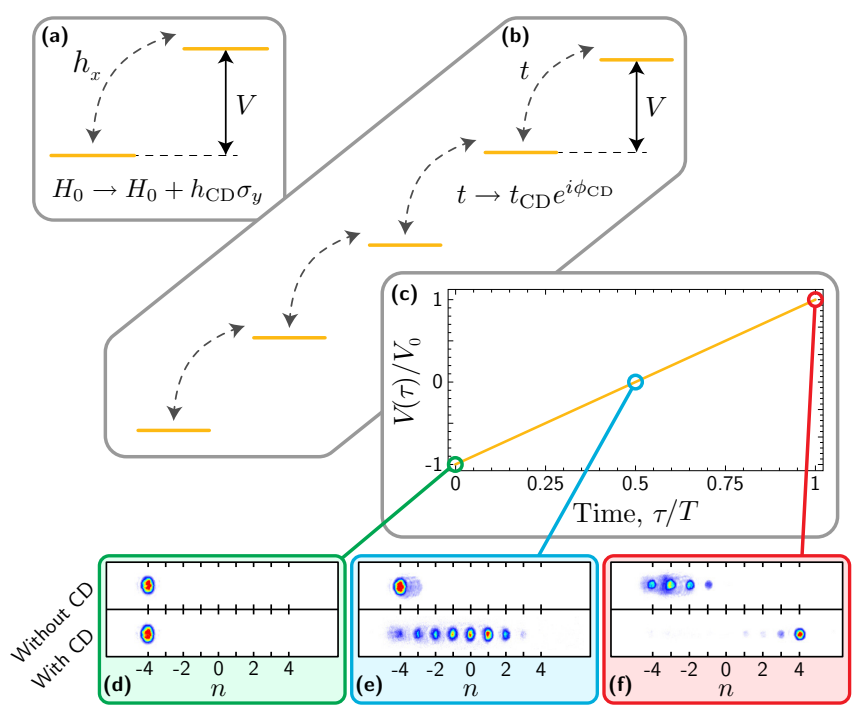

FIG. 1. Counterdiabatic driving in multilevel systems. (a) A two-level representation of counterdiabatic driving. The two levels (yellow lines) are subjected to the Hamiltonian $H_{0}=h_{x} \sigma_{x}+$ $(V(\tau) / 2) \sigma_{z}$. When the site energies are swept in time, however, diabatic terms proportional to $\sigma_{y}$ are introduced in the instantaneous reference frame. (b) An $n$-level representation of counterdiabatic driving. Five sites (yellow lines) in a tight binding model are linked by a constant, real coupling $t$ (dashed black arrows) and all have an energy difference with their neighbors given by the time-dependent function $V(\tau)$. When $V(\tau)$ is changed in time, new effective complex tunneling terms appear. (c) A plot of $V(\tau) / V_{0}$ versus time $\tau / T$ where $V_{0}$ is the maximum energy offset and $T$ is the total ramp duration. (d)-(f) Averaged absorption images for a nine-site lattice undergoing the site-energy ramp shown in panel (c) at three different points during the ramp $\tau=\{0, T / 2, T\}$ shown with green, blue, and red backgrounds respectively. For this data $T=2.5 \mathrm{~ms}, V_{0} / h \approx 4 \mathrm{kHz}$, and $t / h \approx 1 \mathrm{kHz}$. The experiment is performed both without (top images) and with (bottom images) counterdiabatic driving.

driving and topological invariants of the underlying parameter space [30].

Counterdiabatic approaches to surpassing the adiabatic limit are based on actively correcting for diabatic terms that emerge in the instantaneous reference frame when a Hamiltonian is rapidly deformed. The emergence of such terms is illustrated in Fig. 1(a) for a canonical two-level system, having a bare Hamiltonian $H_{0}=h_{x} \sigma_{x}+(V(\tau) / 2) \sigma_{z}$, where the $\sigma_{i}$ are Pauli matrices. Here, $h_{x}$ sets a fixed scale for offdiagonal coupling between the two basis states of the system, and $V(\tau)$ sets the time-dependent diagonal energy difference between the two levels, with time denoted by $\tau$. Considering the well-known adiabatic rapid passage (ARP) protocol depicted in Fig. 1(c), in which the diagonal energy difference $V$ is linearly ramped while the off-diagonal coupling $h_{x}$ is held fixed, a new, effective coupling term $h_{\mathrm{CD}} \sigma_{y}$ will appear in the instantaneous reference frame of the particles. This emergent term can lead to transitions between the instantaneous eigenstates, and can result in a breakdown of the ARP protocol.

In extending this example to the many-level scenario shown in Fig. 1(b), we abandon the Pauli matrices in favor of a formalism that reflects the symmetry of the couplings relevant to a uniform tight-binding lattice. Specifically, the bare Hamiltonian in this case contains off-diagonal coupling between nearest-neighbor states at a scale set by the (realvalued) tunneling energy $t$, along with diagonal site energies that are offset from their nearest neighbors by an amount $V(\tau)$ [as defined later in Eq. (11)]. As in the two-level case, when the energy difference between the neighboring sites is changed dynamically in time, new diabatic terms appear in the instantaneous reference frame. These diabatic terms appear as added contributions to the off-diagonal tunneling that are imaginary, i.e., $\pi / 2$ out of phase with respect to the bare, real tunneling. These imaginary tunneling contributions, direct extensions of the emergent $\sigma_{y}$ term of the two-level case, can be incorporated along with the bare tunneling $t$ into a modified overall tunneling energy $t_{\mathrm{CD}}$ and tunneling phase $\phi_{\mathrm{CD}}$. To note, the appearance of such phases underlies the use of shaken-lattice techniques for the dynamical generation of tunneling phases $[31,32]$. In contrast, the ability to directly engineer tunneling phases in our synthetic lattice platform can allow us to counteract these dynamical modifications of the tunneling phases, and thus enable faster-than-adiabatic population transport and state transformations.

These diabatic terms (the $\sigma_{y}$ term in the two-level system and the modified tunneling energy and tunneling phase in the $n$-level system) can be determined analytically. Thus with proper experimental controls, new terms may be introduced to the Hamiltonian that, when we enter the instantaneous reference frame, cancel with the newly acquired diabatic terms and thus mitigate diabatic transitions between instantaneous eigenstates. This is the idea beyond counterdiabatic (CD) driving, and its ability to overcome the adiabatic limit. We present a lattice example of this effect in Figs. 1(d)-1(f) using ultra cold ${ }^{87} \mathrm{Rb}$ in a synthetic lattice of nine coupled momentum states, which will be described in more detail later in this manuscript. The goal of this experiment is to faithfully transfer population across a multisite lattice (from site $n=1$ to $n=9$ ) in a time that is shorter than needed for adiabatic transport. Our nine-site lattice, with an initial tilt of $V_{0} / h \approx 4 \mathrm{kHz}$ and a final tunneling of $t / h \approx 1 \mathrm{kHz}$, is initialized with all population at its left edge in site $n=1$. The site energies and tunneling strengths are then swept according to Eqs. (10) and (11) appearing later in this manuscript, such that the tilt is inverted in a time $T=2.5 \mathrm{~ms}$. Averaged absorption images are shown of population in the lattice at three different times along the ramp $\{0, T / 2, T\}$ and experiments are performed both without (top images) and with (bottom images) the addition of $\mathrm{CD}$ driving. Both experiments start with identical conditions in Fig. 1(d). By time $T / 2$ shown in Fig. 1(e), however, the $\mathrm{CD}$ experiment is already vastly outpacing the experiment without CD driving. Not much transport has occurred without $\mathrm{CD}$ driving whereas the population is roughly halfway to the other side of the lattice with CD driving. Figure 1(f) shows the final distributions of the two experiments. Without CD driving, population has only moved a few sites from the starting position. In contrast, $\mathrm{CD}$ driving enables the majority of the population to be successfully transported the other side of the lattice. This proof-of-concept experiment shows that $\mathrm{CD}$ driving in this case can provide a powerful shortcut to adiabaticity. 


\section{RESULTS}

We present two basic experiments to demonstrate the power of the CD technique and its applicability for state manipulation and preparation in extended, multi-site systems. First, we explore CD-enhanced population transfer across a multisite lattice. In analogy to ARP protocols $[33,34]$ in twolevel systems, we apply a linear energy bias across the sites of a lattice with open boundaries. By inverting this bias "tilt," we attempt to move population from one end of the lattice to the other with high fidelity. Second, we explore how CD methods can aid in preparing the delocalized eigenstates of a multi-site system. Specifically, for a five-site lattice with open boundaries, we prepare and probe its five energy eigenstates.

For both of these experiments, the system is a tight-binding lattice with a Hamiltonian given by

$$
H_{0}=-\sum_{n} t_{n}\left(c_{n}^{\dagger} c_{n+1}+\text { H.c. }\right)+\sum_{n} V_{n} c_{n}^{\dagger} c_{n},
$$

where $t_{n}$ is the real, positive tunneling energy associated with a particle transitioning between sites $n$ and $n+1, c_{n}^{(\dagger)}$ is the annihilation (creation) operator at site $n$, and $V_{n}$ is the energy of the site $n$. Experimentally, the sites of the system are realized by plane-wave momentum states of Bose-condensed ${ }^{87} \mathrm{Rb}$ atoms, the tunneling elements represent two-photon Bragg transitions driven by applied laser fields, and the site energies are controlled through coordinated detunings of the various Bragg laser fields from their resonance conditions [35,36]. To note, we experimentally achieve independent and timedependent control of all $t_{n}$ and $V_{n}$ terms, which allows for the implementation of $\mathrm{CD}$ driving as we now describe, as well as the initial preparation of atoms at different sites (by moving the lattice links relative to an initially populated zero momentum condensate), as we describe later.

We consider attempting to make an adiabatic change to this Hamiltonian by changing the parameters $V_{n}$ and $t_{n}$ as a function of the external control parameter $\lambda(\tau)$, where $\tau$ represents time, such that they become

$$
\begin{aligned}
V_{n} & \rightarrow V_{n}(\lambda), \\
t_{n} & \rightarrow t_{n}(\lambda) .
\end{aligned}
$$

The parameter $\lambda$ encapsulates the time dependence of the Hamiltonian but could, in principle, be a function of an external field or any other variable. For general time-dependence of the system parameters, the instantaneous eigenstates of the system can become coupled by new, diabatic terms in the Hamiltonian [12-14,27]. For this particular Hamiltonian, the dominant diabatic terms,

$$
A=i \sum_{n} \alpha_{n}\left(c_{n}^{\dagger} c_{n+1}-c_{n+1}^{\dagger} c_{n}\right),
$$

take the form of a local current, where $\alpha_{n}$ can be found by solving a closed set of equations. This system of equations, derived in Appendix A and based on the supplementary information of Ref. [27], is given by

$$
\begin{aligned}
-t_{n} \partial_{\lambda}\left(V_{n+1}-V_{n}\right)= & +\alpha_{n}\left(V_{n+1}-V_{n}\right)^{2} \\
& +\alpha_{n}\left(t_{n+1}^{2}+4 t_{n}^{2}+t_{n-1}^{2}\right) \\
& -3 t_{n}\left(t_{n+1} \alpha_{n+1}+t_{n-1} \alpha_{n-1}\right),
\end{aligned}
$$

where every term is a function of $\lambda$ and so the explicit $(\lambda)$ notation has been suppressed. For simplicity, this set of equations includes only the spatial dependence of the tunneling terms and not the time dependence. For the experiments performed herein, the diabatic effects come almost entirely from the time dependence of the site energies and not the tunneling terms, which only negligibly change the $\alpha_{n}$ values. For a full derivation, including all time dependencies, see Appendix A.

In our experimental implementation of counterdiabatic driving, we directly incorporate these diabatic terms into our engineered Hamiltonian through control of the tunneling amplitudes and tunneling phases as

$$
\begin{aligned}
H_{\mathrm{CD}}(\lambda)= & -\sum_{n}\left(t_{n, \mathrm{CD}}(\lambda) e^{-i \phi_{n, \mathrm{CD}}(\lambda)} c_{n}^{\dagger} c_{n+1}+\text { H.c. }\right) \\
& +\sum_{n} V_{n}(\lambda) c_{n}^{\dagger} c_{n},
\end{aligned}
$$

where the new terms $t_{n, \mathrm{CD}}(\lambda)$ and $\phi_{n, \mathrm{CD}}(\lambda)$ are given by

$$
\begin{aligned}
t_{n}(\lambda) & \rightarrow t_{n, \mathrm{CD}}(\lambda)=\sqrt{t_{n}(\lambda)^{2}+\left(\alpha_{n}(\lambda) \partial_{\tau} \lambda(\tau)\right)^{2}}, \\
\phi_{n} & \rightarrow \phi_{n, \mathrm{CD}}(\lambda)=\arctan \left(\frac{t_{n}(\lambda)}{\alpha_{n}(\lambda) \partial_{\tau} \lambda(\tau)}\right) .
\end{aligned}
$$

While the incorporation of the additional control variable $\lambda$ may seem like an unnecessary extra step, the solution in terms of $\lambda$ instead of $\tau$ allows one to solve Eq. (5) without prior knowledge of the explicit functional form of the time dependence. As long as it is known how $t_{n}$ and $V_{n}$ relate to $\lambda$, the functional form of $\lambda$ can be changed without resolving for the $\alpha_{n}(\lambda)$. The site or $n$ dependence of these diabatic corrections, seen in Eqs. (7) and (8), make this direct form of $\mathrm{CD}$ driving only well matched to certain experimental implementations that allow for local control of state-to-state coupling (tunneling) strengths and phases, such as is found in "synthetic lattices." For implementation in other platforms, there exists a remapping of the diabatic corrections through a gauge transformation that allows them to be applied solely to the site energies. Given our ability to directly control the tunneling strengths and phases in our system, we implement the direct, ungauged version of the CD protocol.

\section{A. Transfer across the lattice}

Our first demonstration of how CD driving can provide a shortcut to adiabaticity is in the context of a multisite ARP. We begin with all the population in the highest excited state of a lattice with a variable total number of sites $L$ as depicted in Fig. 2(a). In the absence of CD driving, we linearly ramp the lattice from a positive tilt to a negative tilt. The highest energy mode of the system goes from initially populating the rightmost site at the start of the ramp, to having nearly full overlap with the left-most site at the ramp's end. The parameter ramp we implement, which has a total duration $T$, has the exact form

$$
\lambda(\tau)=1-\frac{\tau}{T} .
$$

This ramp is incorporated into $t_{n}$ and $V_{n}$ as

$$
\begin{gathered}
t_{n}(\lambda)=t(1.1-\lambda)=t\left(0.1+\frac{\tau}{T}\right), \\
V_{n}(\lambda)=n V_{0} 2(\lambda-1 / 2)=n V_{0}\left(1-\frac{2 \tau}{T}\right),
\end{gathered}
$$




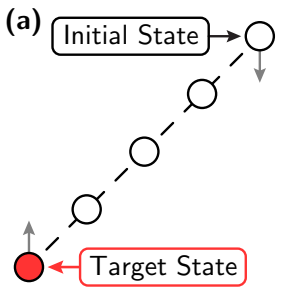

(1)

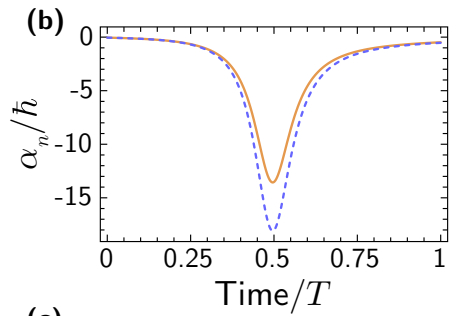

(c)
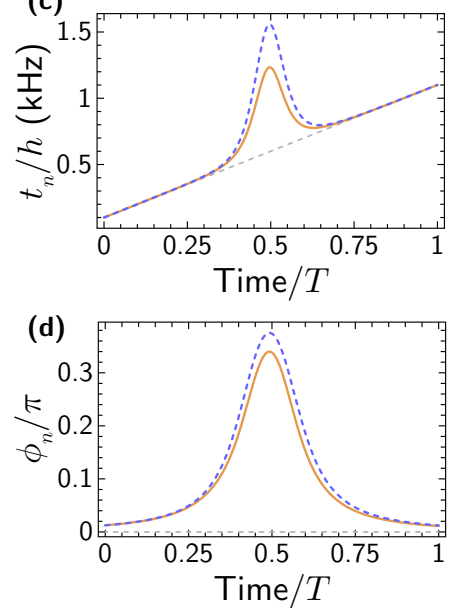
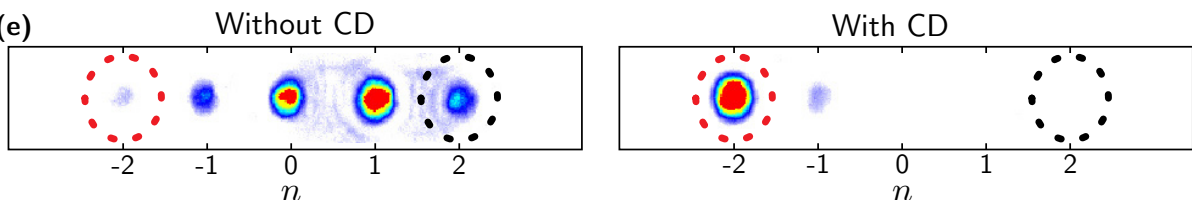

(f)

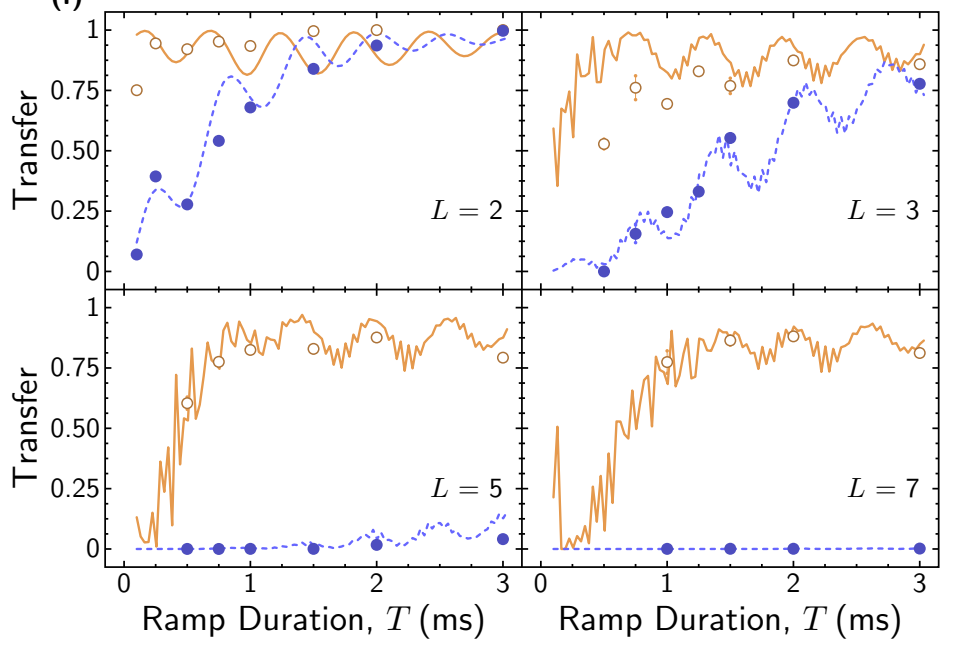

(g)

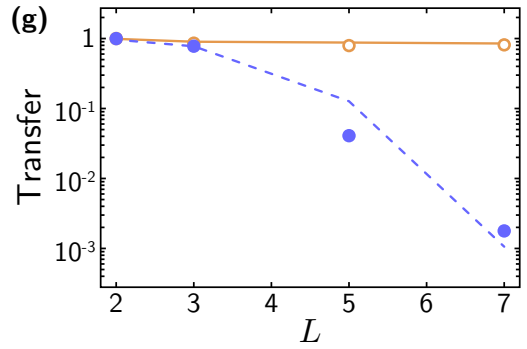

FIG. 2. Enhancement of population transfer by CD driving. (a) Cartoon depiction of the initial, highest-energy state and the target state for data shown in (e). (b)-(d) The $\alpha_{n}, t_{n, \mathrm{CD}}$, and $\phi_{n, \mathrm{CD}}$ for a five site lattice with $n \in\{-2,-1,0,1,2\}$ and $T=2$ ms. The blue dotted line represents the terms for the transitions $-1 \rightarrow 0$ and $0 \rightarrow 1$ and the gold solid line is for $-2 \rightarrow-1$ and $1 \rightarrow 2$. The gray dashed lines in (c) and (d) show $t_{n}$ and $\phi_{n}$ without CD corrections. (e) Averaged (over eight measurements) absorption images for a $T=2 \mathrm{~ms}$ ramp in an $L=5$ site lattice with $V_{0} / h \approx 4 \mathrm{kHz}$ and $t_{n} / h \approx 1 \mathrm{kHz}$. The procedure is performed both without CD driving (left) and with (right). The target state is circled with the red dotted line on the left and the starting state is on the far right circled with the black dotted line. (f) The population transferred from the initial state to the target state at the end of the ramp versus the total ramp duration, $T$. Open orange circles and solid orange lines represent data and simulation when implementing $\mathrm{CD}$ driving during the ramp. Filled blue circles and dashed blue lines represent data and simulation without implementing $\mathrm{CD}$ driving during the ramp. We perform the protocol with $L=2, L=3, L=5$, and $L=7$. (g) Log of population fraction transferred to the target state as a function of system size $L$ for a $T=3 \mathrm{~ms}$ ramp duration. Open orange circles and solid orange line are data and simulation when using CD driving during the ramp. Filled blue circles and dashed blue line are data and simulation without $\mathrm{CD}$ driving during the ramp. The data shown in panels (f) and (g) were taken with a final tunneling $t / h \approx 1 \mathrm{kHz}$ and the simulations, which employ a local density approximation, assume an average mean-field interaction of $U / h=1.25 \mathrm{kHz}$. All error bars in panels (f) and (g) indicate one standard error of the mean.

where $t$ is the characteristic tunneling scale of the lattice at the end of the ramp and $V_{0}$ is the initial site energy slope. With the large energy offset and small tunneling, the eigenstates of the initial Hamiltonian are essentially site localized. The tunneling is independent of $n$, meaning that each link always has the same tunneling strength (in the protocol without CD driving) whereas the site energy is linear with $n$ such that the lattice is tilted with a slope $V_{0}$ at $\tau=0$ and $-V_{0}$ when $\tau=T$. The correction factors $\alpha_{n}$ as calculated from Eq. (5) are plotted along with the modified tunneling strength and phase [from Eqs. (7) and (8)] in Figs. 2(b)-2(d), illustrating the kinds of corrections that can be generated by the CD approach.
The calculated $\alpha_{n}$ values shown in Figs. 2(b)-2(d) are for a five-site lattice undergoing this inversion in a time $T=2 \mathrm{~ms}$ with an initial offset $V_{0} / h \approx 4 \mathrm{kHz}$ and a characteristic tunneling $t / h \approx 1 \mathrm{kHz}$. The $\alpha_{n}$ terms are then used to calculate the CD tunneling strength $t_{n, \mathrm{CD}}(\lambda)$ and phase $\phi_{n, \mathrm{CD}}(\lambda)$. The diabatic terms $\alpha_{n}$ are symmetric about the center of the lattice in this case, such that $\alpha_{-1}=\alpha_{0}$ (blue dashed line) and $\alpha_{-2}=\alpha_{1}$ (solid gold line) as shown in Fig. 2(b). This symmetry propagates onto the tunneling strengths and phases as well, as shown in matching style in Figs. 2(c) and 2(d). The uncorrected tunneling strength and phase are also shown in these figures as gray dashed lines for comparison. 
If performed slowly enough so as to respect adiabaticity conditions, this lattice tilt inversion would move population from the right end of the lattice to the left end, maintaining population of the highest energy eigenstate throughout the evolution. However, if the lattice tilt inversion is performed too rapidly, it can induce non-adiabatic Landau-Zener transitions between the instantaneous eigenstates of the system. For a two-site lattice, corresponding to the textbook ARP, such a breakdown in adiabaticity would result in some population remaining at the initial site. For the many-site system, this breakdown in adiabaticity more generally results in atomic population not making it all the way across the lattice, as a result of undergoing one or more diabatic transitions between the system eigenstates. Figure 2(e) shows this effect in the averaged absorption image data for $L=5, t / h \approx 1 \mathrm{kHz}$, $V_{0} / h \approx 4 \mathrm{kHz}$, and $T=2 \mathrm{~ms}$. Without CD driving (left image in the panel), we detect only a small fraction of atoms in the left target site due to a breakdown in adiabaticity during the lattice inversion. In fact, most atoms remain within one or two sites of the initial position. In contrast, the application of CD driving (right image in the panel) results in nearly all of the atoms transferring to the left-most target site after starting in the right-most site.

We perform this lattice inversion experiment as a function of both ramp duration $T$ and system size $L$ and present the results in Fig. 2(f). To note, we keep $t / h \approx 1 \mathrm{kHz}$ and $V_{0} / h \approx$ $4 \mathrm{kHz}$ fixed as we vary the ramp duration $T$ and system size $L$. On the vertical axis we plot the transfer fraction (the fractional amount of atoms which ended up in the target state) against the ramp duration $T$ on the horizontal axis. Focusing on the $L=2$ case presented in the upper left, we find that implementing CD driving results in a substantial increase in the transferred fraction for shorter ramps as reflected in the data (open orange circles) and simulation (solid orange line) over the case without $\mathrm{CD}$ driving (solid blue dots and dashed blue theory line). Without CD driving, we are eventually able to reach adiabaticity in this small system for ramp durations $T>2 \mathrm{~ms}$. Indeed, in this limit and for just two sites, the diabatic terms become negligible and the $\mathrm{CD}$ and non-CD parameter ramps are nearly identical.

We also perform this lattice transfer experiment as a function of the system size $L$ working with sizes $L=\{2,3,5,7\}$. We observe, unsurprisingly, that without CD driving it becomes increasingly difficult to maintain adiabaticity as the system size grows. This is reflected in Fig. 2(f) by the continued reduction of the transfer fraction for the blue curves/points as $L$ is increased, even out to the longest times explored. With the application of CD driving, however, we are able to transfer $>80 \%$ of population to the target state for all four system sizes on the timescale of just 1-2 ms. For the shortest ramps explored, we observe one practical limitation of the CD driving as applied to our "momentumstate synthetic lattices," which results in a decreased transfer efficiency. Specifically, the CD corrections become quite large for short ramps, and the operational limit of our effective lattice Hamiltonian breaks down as different momentum states become coupled in an off-resonant fashion.

The dependence of the non-CD and CD transfer efficiency on the system size $L$ is summarized in Fig. 2(g). We show the population transferred to the target state as a function of system size for a $T=3 \mathrm{~ms}$ ramp duration for all four system sizes on a semilog scale. We observe that the $\mathrm{CD}$ driving (open orange circles) results in nearly perfect transfer for all lattice sizes, whereas the case without CD driving (solid blue dots) results in a transfer that decreases rapidly with system size, eventually reaching the $10^{-3}$ level for $L=7$. This plot emphasizes the benefits of using $\mathrm{CD}$ driving when performing a nontrivial operation in a system containing an adiabatic limit that scales poorly with system size (i.e., in systems with no adiabatic support in the thermodynamic limit). Such state transfers may be of practical benefit-for example, in this system of coupled momentum states, the measured efficiency of $88 \%$ for transferring across seven synthetic lattice sites in 2 ms relates to an efficiency of over $99 \%$ per imparted recoil momentum ( $\hbar k$, with $k$ the lattice wave vector).

To note, the enormous enhancement in transfer probability found for the largest system size $(L=7)$ is in part due to the fact that the modified tunneling terms of the $C D$ protocol can be larger in amplitude than those of the linear ramp. For the $L=2,3$, and 5 cases in Fig. $2(\mathrm{~g})$, the maximum tunneling amplitudes of the CD protocol do not exceed those of the linear protocol, such that the improvements in these cases can be considered the result of a fair comparison.

\section{B. Preparing and probing eigenstates of few-site lattices}

In addition to performing robust state transformations for practical applications, CD driving also promises to greatly improve the ability to prepare specific wave functions that may be of interest to the study of localization phenomena in synthetic lattices. For example, the physics of topological boundary states $[37,38]$ could be better probed by engineering tailored atomic wave functions that are well matched to boundary modes. In addition, the ability to engineer individual energy eigenstates of certain types of quasiperiodic lattice models could allow for the direct exploration of localization phenomena at a mobility edge [39-43]. Finally, for synthetic lattices of momentum states, as well as for techniques allowing for engineered spin-orbit coupling in atomic gases [44], the physical separation in space of atomic wave packets with distinct momentum can present a practical limitation on the timescales over which coherent nonequilibrium dynamics can be explored. By preparing "dressed" eigenstates (dressed states of the atoms and driving fields) that involve superpositions of multiple different plane-wave momentum states, one can judiciously explore the physics of such systems while circumventing the issue of spatial wave-packet separation.

Here, for demonstration purposes, we consider populating the dressed eigenstates of a simple five-site synthetic lattice by linear ramps with and without $\mathrm{CD}$ driving. Our starting configuration is a five-site lattice with the same initial positive site-energy slope as used in the previous experiment. Here, however, instead of continuing to invert the site energy tilt from positive slope to negative slope, we terminate this procedure at the time when the sites of the lattice all have equal energy values, as illustrated in Fig. 3(a). The form of the parameter ramp function $\lambda(\tau)$, as well as the ramps for the tunneling parameters, are identical to the previous case 

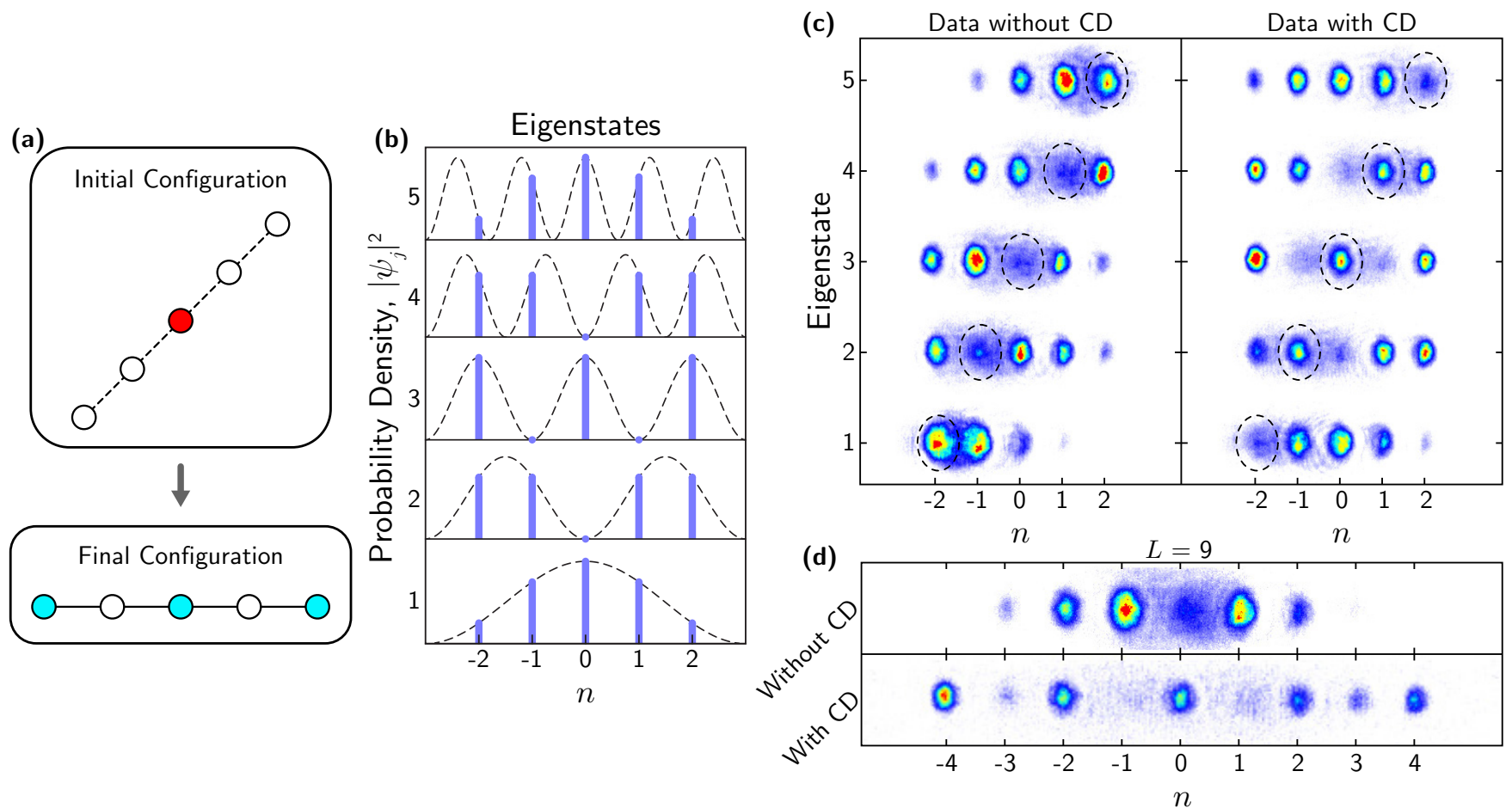

FIG. 3. Preparing the eigenstates of an $L$-site lattice with counterdiabatic driving. (a) Depiction of the ramp protocol for eigenstate preparation. We start in a single site of the initial configuration and the choice of this site dictates which eigenstate is prepared. By smoothly turning off a large gradient, eigenstates of the flat, uniform lattice are prepared. We show as an example how by starting at the central site of the tilted lattice can allow for preparation of the middle-energy eigenstate of a five-site lattice. (b) The probability density for the first five eigenstates of a five-site lattice. The dashed lines indicate the probability for a continuous "infinite square well" potential, whereas the blue bars show the discretized amplitudes relating to a five-site lattice with open boundaries. (c, left) Absorption images for the attempted preparation of all five eigenstates without CD driving. We compare the theoretical probability distribution and our probability distribution without CD driving by calculating the "efficiency" $F_{j}^{\text {adi }}=\{0.722(24), 0.555(11), 0.282(15), 0.619(9), 0.808(7)\}$ for each of the five eigenstates. (c, right) Absorption images for the preparation of all five eigenstates with CD driving during the ramp. For these five states, the associated preparation "efficiencies" are $F_{j}^{\text {cd }}=\{0.983(5), 0.861(5), 0.865(3), 0.911(8), 0.987(2)\}$. Data shown in (c) were taken with a ramp time of $1 \mathrm{~ms}$ and a final tunneling of $t / h=950 \mathrm{~Hz}$. The dashed circles shown for each image in (c) relate to the initial locations of the atomic condensate. Typically, a small residual cloud of thermal atoms remain at this initial location relating to zero momentum. (d) Absorption images showing the attempt to prepare the middle-energy state for a nine-site lattice taken with a ramp time of $1 \mathrm{~ms}$ and a final tunneling of $t / h=1 \mathrm{kHz}$. The upper panel is without $\mathrm{CD}$ driving and the lower panel is with $\mathrm{CD}$ driving.

[i.e., Eqs. (9) and (10)], but now we ramp the site energies as

$$
V_{n}(\lambda)=n V_{0} \lambda=n V_{0}\left(1-\frac{\tau}{T}\right),
$$

where $n$ is the site index, $V_{0}$ is initial slope of the tilted lattice, $T$ is the ramp duration, and $\tau$ is the time variable. The diabatic terms and corrections to the tunneling amplitudes and phases for this experiment look identical to those appearing in the first half of the lattice inversion experiment shown in Figs. 2(b)-2(d). This new ramp results in an initial positive slope of $V_{0}$ at $\tau=0$, but instead of fully inverting, the site energies become equal at $\tau=T$, resulting in a flat and uniform lattice.

The Bloch-like eigenstates of this uniform lattice are essentially just the lowest five eigenstates of a "particle in a box" or infinite square well, however with the wave-function amplitude only appearing at the five discrete positions corresponding to the sites of the lattice, shown in Fig. 3(b). While the higher energy eigenstates should indeed feature more rapid variation of the phase, as is related to the appearance of nodes in the wave functions in the continuous problem, there is a symmetry of the low- and high-energy eigenstates if one looks only at their probability densities. Specifically, as is depicted by the heights of the blue bars in Fig. 3(b), the lowest and highest energy eigenstates (eigenstates 1 and 5) should have equivalent probability density distributions, as should the eigenstates with the second-lowest energy (state 2) and the second-highest energy (state 4).

In the previous experiment (state-transfer by lattice tilt inversion), we specifically loaded the highest-energy eigenstate to start. This was accomplished by starting with all population initialized to our atomic condensate at zero momentum, and then only turning on the appropriate Bragg laser fields that would address transitions to atomic momentum states displaced in one direction (e.g., to states with two photon momenta to the left, four photon momenta to the left, etc.). In general, we have full control over which transitions between momentum states are "turned on" through laser addressing. Physically, we always begin in the zero momentum state, which is denoted by the dashed circles in Fig. 3(c) (and about which one can typically see a small cloud of residual thermal atoms). However, the mapping between physical 
"momentum states" and "synthetic lattice sites" can be modified as described above to allow for the initialization at different sites of the five-site tilted lattice, which in turn allows for the initialization of different eigenstates in the tilted lattice configuration [as depicted in Fig. 3(a)]. In this way we can prepare all five eigenstates $\left|\psi_{j}\right\rangle$ of the uniform open-boundary lattice, first by preparation with an energy bias applied and then by a ramp of the tilt to zero site-to-site bias.

We first attempt this experiment without $C D$ driving, as shown in Fig. 3(c, left) with the parameters $V_{0} / h \approx 4 \mathrm{kHz}$, $t / h \approx 0.95 \mathrm{kHz}$, and $T=1 \mathrm{~ms}$. It is qualitatively clear from the absorption images that the procedure without $\mathrm{CD}$ driving failed to prepare any of the eigenstates, as the probability densities do not match those expected for the eigenstates, as shown in Fig. 3(b). More to the point, the atomic distribution did not spread out much from the initial zero-momentum condensate that was first populated (dashed circles). Quantitatively, we compare the theoretical probability distribution and our probability distribution prepared without $\mathrm{CD}$ driving by calculating the "efficiency" of state preparation. We calculate the efficiency in terms of the normalized number of atoms detected at each lattice site $n$ and for each state $j$ which we define as $P_{n}^{j}$. The "efficiency" $F$ is calculated from these probability distributions by

$$
F_{j}=\left(\sum_{n} \sqrt{P_{n}^{j, \text { theory }} P_{n}^{j, \text { expt }}}\right)^{2}
$$

in terms of the experimental and theoretical probability distributions. In the case without CD driving, $F_{j}^{\text {adi }}=\{0.722(24)$, $0.555(11), 0.282(15), 0.619(9), 0.808(7)\}$ for each of the five eigenstates. To note, this approach works most efficiently for the highest and lowest energy states, which have the fewest neighboring (in energy) eigenstates to which they could undergo diabatic transitions.

We observe that a marked improvement is obtained by applying CD driving to this state preparation protocol, keeping all other parameters the same. We are able to prepare probability distributions which agree well with the desired distributions, with $F_{j}^{\text {cd }}=\{0.983(5), 0.861(5), 0.865(3)$, $0.911(8), 0.987(2)\}$ for eigenstates 1-5, with corresponding experimental absorption images shown in Fig. 3(c, right). Thus the average efficiency for preparing states without CD driving is $0.60(5)$, with an improvement to an average efficiency of $0.92(3)$ via the CD method. Just as for the state transfer across the lattice, this preparation of eigenstates delocalized across the sites can also be extended to larger system sizes. Fig. 3(d) shows (via absorption image) the preparation of the middle-energy eigenstate for an $L=9$ site lattice, which should have the same alternating probability density structure as the middle energy in Fig. 3(b). The upper panel shows the experiment as performed without $\mathrm{CD}$ driving and relates to an efficiency of $\sim 50 \%$. The lower panel, with $\mathrm{CD}$ driving, matches the expected distribution much more closely with an efficiency of $\sim 80 \%$.

These measurements show that we are able to accurately prepare a state that has the same probability density distribution as the desired system eigenstates. However, we have not yet characterized the phase structure of the created wave functions, which is of equal importance. One simple and generic way to test whether we have created the appropriate phase structure for our states (in addition to the probability density distribution) is to simply leave on the base Hamiltonian of a flat lattice with uniform tunnel coupling, and observe whether there is continued evolution of the probability density distribution. If the phase structure is matched to that of the desired eigenstate, then by definition we should see no further dynamics of the population density under that Hamiltonian's evolution. However, any errors in the populations or relative phases between sites can drive currents in the lattice.

Figure 4 summarizes our tests of the phase structure of the prepared states. We perform the same protocol used to prepare the eigenstates of the five-site lattice but with parameters $V_{0} / h \approx 4 \mathrm{kHz}, t / h \approx 900 \mathrm{~Hz}$, and $T=0.75 \mathrm{~ms}$. We attempt to prepare the middle energy eigenstate of the five-site lattice as shown in Fig. 3 both with and without CD driving with the added step of keeping the Hamiltonian on and static after the ramp period for an additional time $T$. Without CD driving, we observe continued dynamics after the end of the ramp as shown in Fig. 4(a). This indicates that we have failed to prepare the eigenstate, which is no surprise since even the probability density of the prepared state did not match the eigenstate at the end of the ramp (black dashed line). In contrast, when we attempt to prepare the eigenstate with the aid of CD driving in Figs. 4(d), we find there is very little dynamics during the hold period after the ramp of the lattice tilt. This clear difference signifies that the $\mathrm{CD}$ protocol results in an improved ability to prepare the desired eigenstate of the Hamiltonian that is left on during the hold period. We further compare the experimental data to simulations based on the implemented protocols, both including and ignoring the influence of atomic interactions. The simulation results with and without interactions for the non-CD scenario are shown in Figs. 4(b) and 4(c), whereas the corresponding simulations for the case of CD driving are shown in Figs. 4(e) and 4(f).

Roughly speaking, both the interacting and non-interacting simulations agree with the qualitative features of the data, showing that the linear ramp leads to dynamics during the hold time, whereas the dynamics are largely absent when CD driving is applied. However, there is additionally a clear, qualitative asymmetry of the population dynamics that is observed in experiment, which is reproduced when interactions are incorporated in the simulations (see Appendix B). Here, the interactions are treated at the mean-field level, with an effectively attractive Hartree term shifting the chemical potential of the populated site [45]. The combination of the initial positive energy tilt and the negative interaction shifts break the symmetry of the population dynamics, as seen in panels (a), (b) for no CD driving and panels (d), (e) for the case of $\mathrm{CD}$ driving. Specifically, as the positive lattice tilt is ramped to zero the effectively negative chemical potential shift of the $n=0$ site due to interactions bring it into resonance with the $n=-1$ site before the end of the ramp at time $T$. This causes population to first flow out to the negative lattice sites, and more generally results in an asymmetry of the population dynamics. In contrast, the dynamics are perfectly symmetric with respect to $n=0$ for the simulations without interactions, shown in panels (c), (f). 


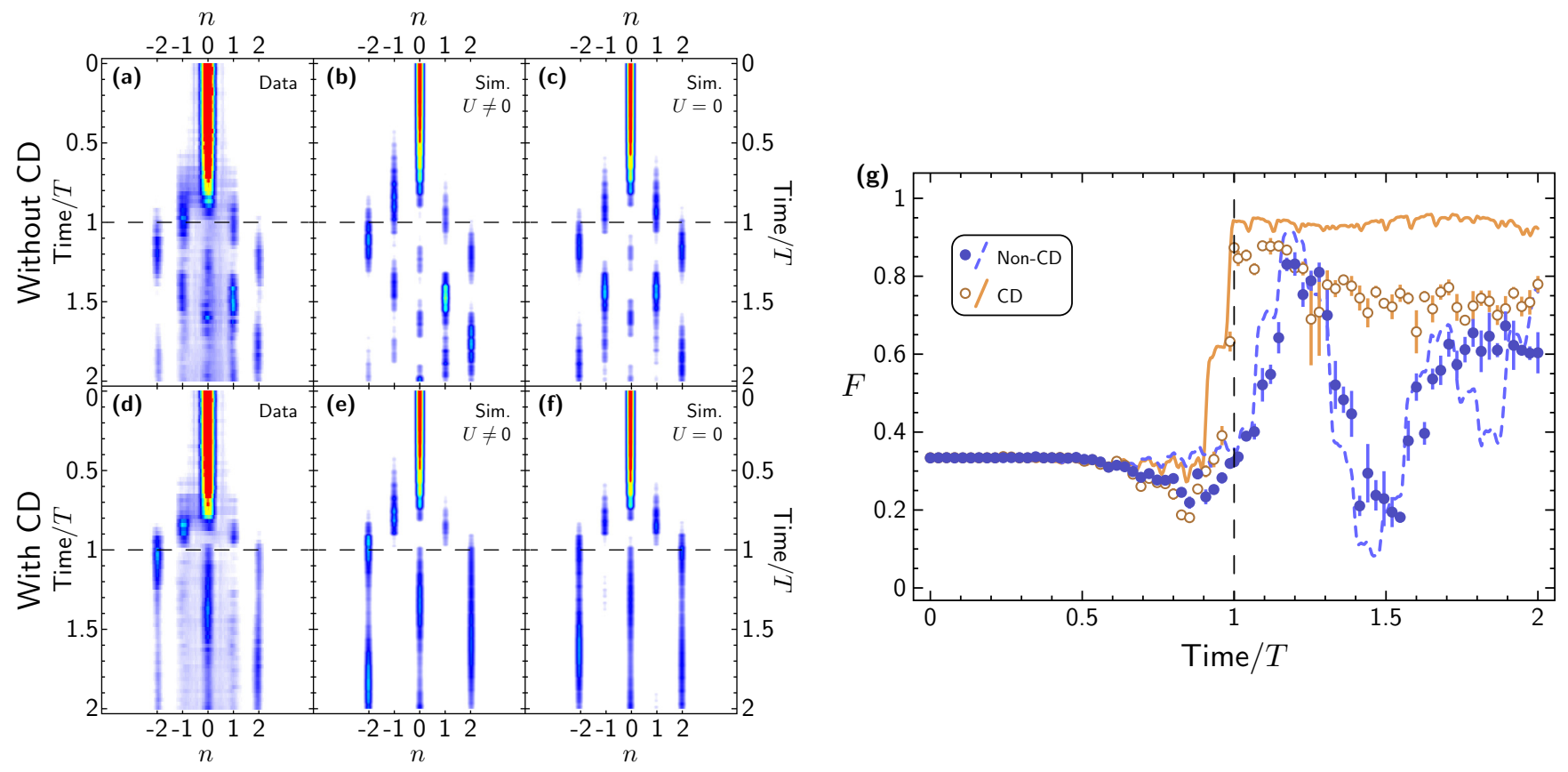

FIG. 4. Testing the phase structure of the states prepared via counterdiabatic driving. Observations of any dynamics after the ramp when preparing the middle-energy state of a five-site lattice for ramps without CD driving [data in (a), simulation with a homogeneous interaction of $U / h=1.25 \mathrm{kHz}$ in (b), and simulation without interactions in (c)] and for ramps with CD driving [data in (c), simulation with a homogeneous interaction of $U / h=1.25 \mathrm{kHz}$ in (d), and simulation without interactions in (e)]. The horizontal dashed lines in all six panels indicate the end of the ramp at time $T$. (g) The resultant efficiency $F$ of the data and simulations with interactions from panels (a), (b), (d), and (e). Data with the $\mathrm{CD}$ driving (open orange circles) is shown with accompanying simulation (orange line) including interactions and off-resonant driving. Data without the CD driving (solid blue circles) is shown with accompanying simulation (dashed blue line) including interactions and off-resonant driving. The vertical dashed line indicates the end of the ramp at time $T$. The data shown here were taken with a ramp time $T=0.75 \mathrm{~ms}$ and a final tunneling $t / h=900 \mathrm{~Hz}$. The simulations in (b), (d), and (e) assume a homogeneous mean-field interaction with strength $U / h=1.25 \mathrm{kHz}$. All error bars in panel (e) represent one standard error of the mean.

The qualitative behavior observed in the density plots of Figs. 4(a)-4(f) are further confirmed by calculating the efficiency $F$ of the ramp in preparing the target state as a function of time $\tau$ throughout the ramp, as shown in Fig. 4(g). Here we calculate $F$ using the target state $P_{n}^{3}=\{1 / 3,0,1 / 3,0,1 / 3\}$ at each snapshot taken during the ramp duration (and with a higher sampling rate for the simulation curves). We confirm our assessment of the raw data by observing extreme oscillations in the efficiency after the end of the ramp for the case without $\mathrm{CD}$ driving and a nearly static, high efficiency for the ramp with $\mathrm{CD}$ driving. The behavior of the $\mathrm{CD}$ driving data reveals that we have nearly correctly prepared the desired eigenstate in both the probability amplitude and phase structure. In contrast, while the non-CD data does reach a high efficiency at a time of roughly $1.2 T$, relating to a nearly ideal density distribution, the ensuing dynamics reveals an error in the generated phase structure.

\section{DISCUSSION}

We have demonstrated that $\mathrm{CD}$ driving can lead to significant improvements over simpler linear schemes in relation to population transfer and state preparation in multilevel, latticelike systems. We showed that the CD approach can improve the preparation of system eigenstates, which will aid in exploration of tight-binding models with novel eigenstates, as well as the engineering of laser-"dressed" atomic momentum states. These experiments represent the first implementation of a truly non-trivial shortcut to adiabaticity protocol, i.e., in a system beyond just two or three states that does not possess scaling symmetry. As such they prove the power of this technique to transform states in systems with small gaps or poor adiabatic scaling. Indeed, this regime of many-level systems is where the use of these shortcuts yields the largest improvements over adiabatic protocols. The specific fast, robust, and efficient manipulations we have explored promise to provide practical advantages for applications such as atom interferometry, and to enable new explorations of exotic phenomena related to topology and disorder in synthetic lattices. By incorporating controlled loss in our synthetic lattice $[46,47]$, such approaches may also allow for unique explorations into fasterthan-adiabatic approaches in non-Hermitian systems [48].

In this work, we have restricted our explorations to the regime in which the lattice bandwidth (four times the tunneling energy) was much larger than the mean-field interaction energy. As such, the mean-field interactions in the system result in small quantitative changes to the simulation curves in Fig. 2 (see Appendix B) and some small qualitative differences in the dynamical behavior observed in Fig. 4. However, for smaller tunneling values the native atomic interactions can drastically alter the behavior of this system [45]. One straightforward extension would be to harness these interactions to investigate optimal $\mathrm{CD}$ driving protocols in the presence of strong nonlinearities [49-53]. 
The combination of native interactions in our system and the ability to explore CD driving may also enable future explorations of topological defects in interacting analog systems. Specifically, there exists a formal connection between the diabatic errors that are generated by the change of a Hamiltonian and the Berry curvature of the underlying Hamiltonian parameter space [30]. This connection has enabled previous measurements of Chern numbers for noninteracting Abelian $[30,54]$ and non-Abelian $[55,56]$ analog systems based on superconducting qubits and cold atoms, as well as explorations into how interactions enrich this problem for an interacting pair of qubits [57]. The interactions in our system could allow for natural extensions of these results, enabling investigations of interaction-stretched monopoles [28] and emergent Yang monopoles [29] in a cold atom simulator.

\section{ACKNOWLEDGMENTS}

This material is based upon work (E.J.M. and B.G.) supported by the Air Force Office of Scientific Research under Grant No. FA9550-18-1-0082. The authors thank A. Polkovnikov for insightful discussions. D.S. acknowledges support of the Research Foundation Flanders (FWO) under Grant No. 12M1518N.

\section{APPENDIX A: DERIVATION OF COUNTER-DIABATIC EQUATIONS}

\section{Time-dependent energy and spatial-dependent tunneling}

Here we derive the optimal local counterdiabatic gauge for transitionless driving of a general free fermion problem. We will be focus on the Hamiltonians of the form

$$
H=-\sum_{j} t_{j}\left(c_{j}^{\dagger} c_{j+1}+\text { H.c. }\right)+\sum_{j} V_{j}(\lambda) c_{j}^{\dagger} c_{j},
$$

where $c_{j}^{\dagger}$ creates a fermion on site $j$ and $c_{j}$ annihilates the fermion. The approximate adiabatic gauge potential defining $\mathrm{CD}$ driving should minimize the following action (see Ref. [27]):

$$
\mathcal{S}\left(\mathcal{A}_{\lambda}\right)=\frac{\operatorname{Tr}\left[G_{\lambda}^{2}\right]}{\operatorname{Tr} \mathbb{I}},
$$

where

$$
G_{\lambda}=\partial_{\lambda} H+i\left[\mathcal{A}_{\lambda}, H\right] .
$$

For quadratic problems the adiabatic gauge potential is also quadratic. Because it is also imaginary it has to be expressed in the form:

$$
\mathcal{A}_{\lambda}=i \sum_{j, k} \alpha_{j, k}\left(c_{k}^{\dagger} c_{j}-\text { H.c. }\right)
$$

where $\alpha_{j, k}=-\alpha_{k, j}$ and all elements are real. Here, we are not concerned with finding the exact adiabatic gauge potentials but rather in their best local approximations, as this drive can be implemented in the experiment by appropriately tuning the amplitude and phase of the lasers. Thus, as before, we are restricting $\mathcal{A}_{\lambda}$ to the following form:

$$
\mathcal{A}_{\lambda}=i \sum_{j} \alpha_{j}\left(c_{j}^{\dagger} c_{j+1}-\text { H.c. }\right),
$$

where the $\alpha_{j}$ 's remain to be determined by minimizing Eq. (A2). It is straightforward to check that

$$
\begin{aligned}
G_{\lambda}= & \sum_{j}\left(\partial_{\lambda} V_{j}-2\left(t_{j} \alpha_{j}-t_{j-1} \alpha_{j-1}\right)\right) c_{j}^{\dagger} c_{j} \\
& +\sum_{j} \alpha_{j}\left(V_{j}-V_{j+1}\right)\left(c_{j+1}^{\dagger} c_{j}+c_{j}^{\dagger} c_{j+1}\right) \\
& +\sum_{j}\left(t_{j} \alpha_{j+1}-\alpha_{j} t_{j+1}\right)\left(c_{j+2}^{\dagger} c_{j}+c_{j}^{\dagger} c_{j+2}\right) .
\end{aligned}
$$

Up to the terms independent of $\mathcal{A}_{\lambda}$, it follows from, e.g., Wick's theorem that the action (A2) is simply proportional to the sum of squares of the individual contributions in the expression above:

$$
\begin{aligned}
\mathcal{S}\left(\mathcal{A}_{\lambda}\right)= & \text { const }+\frac{1}{4} \sum_{j}\left(\partial_{\lambda} V_{j}-2\left(t_{j} \alpha_{j}-t_{j-1} \alpha_{j-1}\right)\right)^{2} \\
& +\frac{1}{2} \sum_{j}\left[\left(t_{j} \alpha_{j+1}-\alpha_{j} t_{j+1}\right)^{2}+\left(V_{j+1}-V_{j}\right)^{2} \alpha_{j}^{2}\right] .
\end{aligned}
$$

Minimizing the action with respect to $\alpha_{j}$ yields the following set of linear equations:

$$
\begin{aligned}
& -3\left(t_{j} t_{j+1}\right) \alpha_{j+1}+\left(t_{j-1}^{2}+4 t_{j}^{2}+t_{j+1}^{2}\right) \alpha_{j}-3\left(t_{j} t_{j-1}\right) \alpha_{j-1} \\
& +\left(V_{j+1}-V_{j}\right)^{2} \alpha_{j}=-t_{j}\left(\partial_{\lambda} V_{j+1}-\partial_{\lambda} V_{j}\right)
\end{aligned}
$$

In some specific cases these equations can be solved analytically but in general one has to resort to numerical methods.

\section{Time-dependent energy and spatiotemporal-dependent tunneling}

One can go through exactly the same exercise as in the previous section, but for a time-dependent tunneling rather than a time dependent potential. Because of the linearity of a problem, a general counterdiabatic drive for time-dependent tunneling and potential will simply be the sum of two independent drives. Consider,

$$
H=-\sum_{j} t_{j}(\lambda)\left(c_{j}^{\dagger} c_{j+1}+\text { H.c. }\right)+\sum_{j} V_{j} c_{j}^{\dagger} c_{j} .
$$

In order to compute the action (A2), we can recycle most of our previous results. We simply need to replace a few terms in expression (A6), resulting in

$$
\begin{aligned}
G_{\lambda}=-2 & \sum_{j}\left(t_{j} \alpha_{j}-t_{j-1} \alpha_{j-1}\right) c_{j}^{\dagger} c_{j} \\
& +\sum_{j}\left[-\partial_{\lambda} t_{j}+\alpha_{j}\left(V_{j}-V_{j+1}\right)\right]\left(c_{j+1}^{\dagger} c_{j}+c_{j}^{\dagger} c_{j+1}\right) \\
& +\sum_{j}\left(t_{j} \alpha_{j+1}-\alpha_{j} t_{j+1}\right)\left(c_{j+2}^{\dagger} c_{j}+c_{j}^{\dagger} c_{j+2}\right) . \quad(\mathrm{A} 10)
\end{aligned}
$$


Such that the action reads

$$
\begin{aligned}
\mathcal{S}\left(\mathcal{A}_{\lambda}\right)= & \text { const }+\sum_{j}\left(t_{j} \alpha_{j}-t_{j-1} \alpha_{j-1}\right)^{2} \\
& +\frac{1}{2} \sum_{j}\left(t_{j} \alpha_{j+1}-\alpha_{j} t_{j+1}\right)^{2} \\
& +\frac{1}{2} \sum_{j}\left(\partial_{\lambda} t_{j}+\left(V_{j+1}-V_{j}\right) \alpha_{j}\right)^{2} .
\end{aligned}
$$

Minimizing this action with respect to $\alpha_{j}$ yields the following set of linear equations:

$$
\begin{aligned}
& -3\left(t_{j} t_{j+1}\right) \alpha_{j+1}+\left(t_{j-1}^{2}+4 t_{j}^{2}+t_{j+1}^{2}\right) \alpha_{j}-3\left(t_{j} t_{j-1}\right) \alpha_{j-1} \\
& +\left(V_{j+1}-V_{j}\right)^{2} \alpha_{j}=-\partial_{\lambda} t_{j}\left(V_{j+1}-V_{j}\right) .
\end{aligned}
$$

This expression ought to be compared with (A8) for the gauge potential of time-dependent potential.

\section{APPENDIX B: MEAN-FIELD SIMULATIONS OF ATOMIC TRANSPORT}

While atomic interactions can play a nontrivial role in the dynamics of atoms in the momentum-space lattice (as demonstrated in Ref. [45]), for these experiments we operate in a regime in which the interactions do not drastically alter the results. However we do find small quantitative effects, such as a slight asymmetry of the amount of transfer across the lattice in the "lattice inversion experiment" with respect to a positive or negative sweep of the lattice tilt, or small differences in the widths of the high and low-energy eigenstates in the "eigenstate preparation experiment" (which should be equivalent in the absence of interactions). These interaction effects were small, however, such that they did not lead to drastic deviations from the single-particle behavior. Still, the calculated theory curves in Fig. 2(f) and 2(g) take atomic interactions into account based on mean-field, Gross-Pitaevskii equation (GPE) calculations. These GPE calculations, which include interactions at roughly the same scale as the maximum tunneling strengths, provide an improved agreement with the observed "lattice transfer" data. In Fig. 5 we show a replication of Fig. 2(f) with a comparison of the mean-field simulations and noninteracting simulations present. We find that the simulations more-or-less qualitatively agree, whereas the simulations with interactions fit the non-CD data slightly better (especially at early ramp durations for $L=2$ and 3 ).

One clear qualitative impact of the interactions is observed in Fig. 4, in which we attempt to prepare the middle energy eigenstate of an $L=5$ site lattice. Interaction shifts of the site energies break the symmetry of the problem, and lead population to first flow to negative sites from $n=0$, and more generally lead to an asymmetry of the population dynamics. The role of interactions is confirmed by the favorable comparison of the data with the simulations that include interactions, as well as the disagreement with the noninteracting simulations.

The interactions in our system of ultracold neutral atoms relate to short-ranged contact interactions in real space. In momentum space, these relate to long-ranged or all-to-all interactions between atoms occupying the discrete momentum

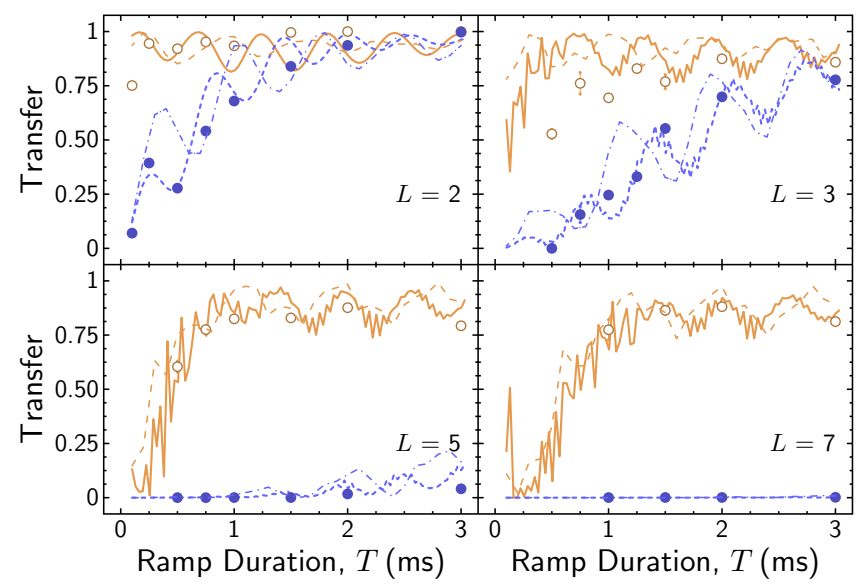

FIG. 5. The population transferred from the initial state to the target state at the end of the ramp versus the total ramp duration, $T$. Open orange circles and solid orange lines represent data and simulation (with average mean-field interaction of $U / h=1.25 \mathrm{kHz}$ ) when implementing $\mathrm{CD}$ driving during the ramp. Filled blue circles and dashed blue lines represent data and simulation (with average mean-field interaction of $U / h=1.25 \mathrm{kHz}$ ) without implementing $\mathrm{CD}$ driving during the ramp. Dashed orange lines and dash-dotted blue lines represent simulations assuming a noninteracting system. We perform the protocol with $L=2, L=3, L=5$, and $L=7$.

orders. If these interactions were simply isotropic, or modeindependent, then the atomic interactions would be incapable of driving any correlated behavior, as the total interaction energy would be a simple constant of motion. Indeed, the direct interactions between pairs of atoms occupying any set of momentum modes are independent of the mode occupation. However, there is an additional exchange energy for pairs of our identical bosonic atoms that interact while occupying distinguishable momentum states [58]. This added intermode interaction (repulsive in our case of having a positive scattering length for ${ }^{87} \mathrm{Rb}$ ), which is a consequence of bosonic statistics, i.e., the symmetry of the two-boson wave function, relates to an effectively attractive interaction between pairs of atoms occupying the same momentum mode [45].

We model the particle dynamics within the synthetic lattice by the following mean-field equation:

$$
i \partial_{\tau} \psi(\tau)=H \psi(\tau)-U|\psi(\tau)|^{2} \psi(\tau),
$$

where $H$ is the single-particle tight-binding Hamiltonian (including any $\mathrm{CD}$ driving terms), $\psi(\tau)$ is the wave function with $\tau$ for time, and $U$ is the average mean-field interaction strength with the negative sign indicating an effectively attractive interaction. The atomic density of the BEC is not uniform but follows a Thomas-Fermi profile and therefore the meanfield strength should also be non-uniform. We account for this by taking a local density approximation where the simulation is split into shells of roughly equivalent density and therefore equivalent mean-field interaction energy. These local simulations are then recombined by weighted averaging to yield the final result. In the case of Figs. 2(f) and 2(g), the simulations were broken up into 20 shells based on an average mean-field interaction of $U / h=1.25 \mathrm{kHz}$. For the simulations of Fig. 4, a homogeneous interaction of $U / h=1.25 \mathrm{kHz}$ is assumed (i.e., no local density approximation is employed in that case). 
[1] D. Guéry-Odelin, A. Ruschhaupt, A. Kiely, E. Torrontegui, S. Martínez-Garaot, and J. G. Muga, Shortcuts to adiabaticity: Concepts, methods, and applications, Rev. Mod. Phys. 91, 045001 (2019).

[2] T. Lausch, M. Hohmann, F. Kindermann, D. Mayer, F. Schmidt, and A. Widera, Optimizing quantum gas production by an evolutionary algorithm, Appl. Phys. B 122, 112 (2016).

[3] A. J. Barker, H. Style, K. Luksch, S. Sunami, D. Garrick, F. Hill, C. J. Foot, and E. Bentine, Applying machine learning optimization methods to the production of a quantum gas, Mach. Learn.: Sci. Technol. 1, 015007 (2020).

[4] J. H. M. Jensen, M. Gajdacz, S. Z. Ahmed, J. H. Czarkowski, C. Weidner, J. Rafner, J. J. Sørensen, K. Mølmer, and J. F. Sherson, Crowdsourcing human common sense for quantum control, arXiv:2004.03296.

[5] D. Sels, Stochastic gradient ascent outperforms gamers in the quantum moves game, Phys. Rev. A 97, 040302(R) (2018).

[6] N. Khaneja, T. Reiss, C. Kehlet, T. Schulte-Herbrüggen, and S. J. Glaser, Optimal control of coupled spin dynamics: design of NMR pulse sequences by gradient ascent algorithms, J. Magn. Reson. 172, 296 (2005).

[7] P. Doria, T. Calarco, and S. Montangero, Optimal Control Technique for Many-Body Quantum Dynamics, Phys. Rev. Lett. 106, 190501 (2011)

[8] A. Omran, H. Levine, A. Keesling, G. Semeghini, T. T. Wang, S. Ebadi, H. Bernien, A. S. Zibrov, H. Pichler, S. Choi, J. Cui, M. Rossignolo, P. Rembold, S. Montangero, T. Calarco, M. Endres, M. Greiner, V. Vuletić, and M. D. Lukin, Generation and manipulation of Schrödinger cat states in Rydberg atom arrays, Science 365, 570 (2019)

[9] E. Farhi, J. Goldstone, and S. Gutmann, A quantum approximate optimization algorithm, arXiv:1411.4028.

[10] G. Pagano, A. Bapat, P. Becker, K. S. Collins, A. De, P. W. Hess, H. B. Kaplan, A. Kyprianidis, W. L. Tan, C. Baldwin, L. T. Brady, A. Deshpande, F. Liu, S. Jordan, A. V. Gorshkov, and C. Monroe, Quantum approximate optimization of the longrange Ising model with a trapped-ion quantum simulator, Proc. Natl. Acad. Sci. USA 117, 25396 (2020).

[11] E. Torrontegui, S. Ibáñez, S. Martínez-Garaot, M. Modugno, A. del Campo, D. Guéry-Odelin, A. Ruschhaupt, X. Chen, and J. G. Muga, Chapter 2 - Shortcuts to adiabaticity, Adv. At. Mol. Opt. Phys. 62, 117 (2013).

[12] M. Demirplak and S. A. Rice, Adiabatic population transfer with control fields, J. Phys. Chem. A 107, 9937 (2003).

[13] M. Demirplak and S. A. Rice, Assisted adiabatic passage revisited, J. Phys. Chem. B 109, 6838 (2005).

[14] M. V. Berry, Transitionless quantum driving, J. Phys. A Math. Theor. 42, 365303 (2009).

[15] X. Chen, I. Lizuain, A. Ruschhaupt, D. Guéry-Odelin, and J. G. Muga, Shortcut to Adiabatic Passage in Two- and Three-Level Atoms, Phys. Rev. Lett. 105, 123003 (2010).

[16] A. Sala, D. L. Núñez, J. Martorell, L. De Sarlo, T. Zibold, F. Gerbier, A. Polls, and B. Juliá-Díaz, Shortcut to adiabaticity in spinor condensates, Phys. Rev. A 94, 043623 (2016).

[17] A. del Campo, Shortcuts to Adiabaticity by Counterdiabatic Driving, Phys. Rev. Lett. 111, 100502 (2013).

[18] S. Deffner, C. Jarzynski, and A. del Campo, Classical and Quantum Shortcuts to Adiabaticity for Scale-Invariant Driving, Phys. Rev. X 4, 021013 (2014).
[19] S. Deng, A. Chenu, P. Diao, F. Li, S. Yu, I. Coulamy, A. del Campo, and $\mathrm{H}$. Wu, Superadiabatic quantum friction suppression in finite-time thermodynamics, Sci. Adv. 4, eaar5909 (2018).

[20] M. G. Bason, M. Viteau, N. Malossi, P. Huillery, E. Arimondo, D. Ciampini, R. Fazio, V. Giovannetti, R. Mannella, and O. Morsch, High-fidelity quantum driving, Nat. Phys. 8, 147 (2012).

[21] J. Zhang, J. H. Shim, I. Niemeyer, T. Taniguchi, T. Teraji, H. Abe, S. Onoda, T. Yamamoto, T. Ohshima, J. Isoya, and D. Suter, Experimental Implementation of Assisted Quantum Adiabatic Passage in a Single Spin, Phys. Rev. Lett. 110, 240501 (2013).

[22] Z. Zhang, T. Wang, L. Xiang, J. Yao, J. Wu, and Y. Yin, Measuring the Berry phase in a superconducting phase qubit by a shortcut to adiabaticity, Phys. Rev. A 95, 042345 (2017).

[23] Z. Zhang, T. Wang, L. Xiang, Z. Jia, P. Duan, W. Cai, Z. Zhan, Z. Zong, J. Wu, L. Sun, Y. Yin, and G. Guo, Experimental demonstration of work fluctuations along a shortcut to adiabaticity with a superconducting Xmon qubit, New J. Phys. 20, 085001 (2018).

[24] T. Wang, Z. Zhang, L. Xiang, Z. Jia, P. Duan, W. Cai, Z. Gong, Z. Zong, M. Wu, J. Wu, L. Sun, Y. Yin, and G. Guo, The experimental realization of high-fidelity 'shortcut-to-adiabaticity' quantum gates in a superconducting Xmon qubit, New J. Phys. 20, 065003 (2018).

[25] B. Zhou, A. Baksic, H. Ribeiro, C. Yale, F. Heremans, P. Jerger, A. Auer, G. Burkard, A. Clerk, and D. Awschalom, Accelerated quantum control using superadiabatic dynamics in a solid-state lambda system, Nat. Phys. 13, 330 (2016).

[26] A. Vepsäläinen, S. Danilin, and G. S. Paraoanu, Superadiabatic population transfer in a three-level superconducting circuit, Sci. Adv. 5, eaau5999 (2019).

[27] D. Sels and A. Polkovnikov, Minimizing irreversible losses in quantum systems by local counterdiabatic driving, Proc. Natl. Acad. Sci. USA 114, E3909 (2017).

[28] T.-L. Ho and C. Li, The Chern numbers of interaction-stretched monopoles in spinor Bose condensates, arXiv:1704.03833.

[29] Y. Yan and Q. Zhou, Yang Monopoles and Emergent ThreeDimensional Topological Defects in Interacting Bosons, Phys. Rev. Lett. 120, 235302 (2018).

[30] V. Gritsev and A. Polkovnikov, Dynamical quantum Hall effect in the parameter space, Proc. Natl. Acad. Sci. USA 109, 6457 (2012).

[31] J. Struck, C. Ölschläger, M. Weinberg, P. Hauke, J. Simonet, A. Eckardt, M. Lewenstein, K. Sengstock, and P. Windpassinger, Tunable Gauge Potential for Neutral and Spinless Particles in Driven Optical Lattices, Phys. Rev. Lett. 108, 225304 (2012).

[32] J. Struck, C. Ölschläger, R. Le Targat, P. Soltan-Panahi, A. Eckardt, M. Lewenstein, P. Windpassinger, and K. Sengstock, Quantum simulation of frustrated classical magnetism in triangular optical lattices, Science 333, 996 (2011).

[33] C. Zener and R. H. Fowler, Non-adiabatic crossing of energy levels, Proc. R. Soc. London, Ser. A 137, 696 (1932).

[34] E. Peik, M. Ben Dahan, I. Bouchoule, Y. Castin, and C. Salomon, Bloch oscillations of atoms, adiabatic rapid passage, and monokinetic atomic beams, Phys. Rev. A 55, 2989 (1997).

[35] B. Gadway, Atom-optics approach to studying transport phenomena, Phys. Rev. A 92, 043606 (2015). 
[36] E. J. Meier, F. A. An, and B. Gadway, Atom-optics simulator of lattice transport phenomena, Phys. Rev. A 93, 051602(R) (2016).

[37] E. J. Meier, F. A. An, and B. Gadway, Observation of the topological soliton state in the Su-Schrieffer-Heeger model, Nat. Commun. 7, 13986 (2016).

[38] E. J. Meier, F. A. An, A. Dauphin, M. Maffei, P. Massignan, T. L. Hughes, and B. Gadway, Observation of the topological Anderson insulator in disordered atomic wires, Science 362, 929 (2018).

[39] S. Ganeshan, J. H. Pixley, and S. Das Sarma, Nearest Neighbor Tight Binding Models with an Exact Mobility Edge in One Dimension, Phys. Rev. Lett. 114, 146601 (2015).

[40] J. Biddle, B. Wang, D. J. Priour, and S. Das Sarma, Localization in one-dimensional incommensurate lattices beyond the Aubry-André model, Phys. Rev. A 80, 021603(R) (2009).

[41] J. Biddle and S. Das Sarma, Predicted Mobility Edges in One-Dimensional Incommensurate Optical Lattices: An Exactly Solvable Model of Anderson Localization, Phys. Rev. Lett. 104, 070601 (2010).

[42] F. A. An, E. J. Meier, and B. Gadway, Engineering a FluxDependent Mobility Edge in Disordered Zigzag Chains, Phys. Rev. X 8, 031045 (2018).

[43] H. P. Lüschen, S. Scherg, T. Kohlert, M. Schreiber, P. Bordia, X. Li, S. Das Sarma, and I. Bloch, Single-Particle Mobility Edge in a One-Dimensional Quasiperiodic Optical Lattice, Phys. Rev. Lett. 120, 160404 (2018).

[44] V. Galitski and I. B. Spielman, Spin-orbit coupling in quantum gases, Nature (London) 494, 49 (2013).

[45] F. A. An, E. J. Meier, J. Ang'ong'a, and B. Gadway, Correlated Dynamics in a Synthetic Lattice of Momentum States, Phys. Rev. Lett. 120, 040407 (2018).

[46] S. Lapp, J. Ang'ong'a, F. A. An, and B. Gadway, Engineering tunable local loss in a synthetic lattice of momentum states, New J. Phys. 21, 045006 (2019).

[47] W. Gou, T. Chen, D. Xie, T. Xiao, T.-S. Deng, B. Gadway, W. Yi, and B. Yan, Tunable Nonreciprocal
Quantum Transport through a Dissipative Aharonov-Bohm Ring in Ultracold Atoms, Phys. Rev. Lett. 124, 070402 (2020).

[48] S. Ibáñez, S. Martínez-Garaot, X. Chen, E. Torrontegui, and J. G. Muga, Shortcuts to adiabaticity for non-Hermitian systems, Phys. Rev. A 84, 023415 (2011).

[49] H. Saberi, T. Opatrný, K. Mølmer, and A. del Campo, Adiabatic tracking of quantum many-body dynamics, Phys. Rev. A 90, 060301(R) (2014).

[50] S. Campbell, G. De Chiara, M. Paternostro, G. M. Palma, and R. Fazio, Shortcut to Adiabaticity in the Lipkin-Meshkov-Glick Model, Phys. Rev. Lett. 114, 177206 (2015).

[51] M. Bukov, D. Sels, and A. Polkovnikov, Geometric Speed Limit of Accessible Many-Body State Preparation, Phys. Rev. X 9, 011034 (2019).

[52] T. Hatomura, Shortcuts to adiabaticity in the infinite-range Ising model by mean-field counter-diabatic driving, J. Phys. Soc. Japan 86, 094002 (2017).

[53] T. Hatomura, Shortcuts to adiabatic cat-state generation in bosonic Josephson junctions, New J. Phys. 20, 015010 (2018).

[54] M. D. Schroer, M. H. Kolodrubetz, W. F. Kindel, M. Sandberg, J. Gao, M. R. Vissers, D. P. Pappas, A. Polkovnikov, and K. W. Lehnert, Measuring a Topological Transition in an Artificial Spin-1/2 System, Phys. Rev. Lett. 113, 050402 (2014).

[55] M. Kolodrubetz, Measuring the Second Chern Number from Nonadiabatic Effects, Phys. Rev. Lett. 117, 015301 (2016).

[56] S. Sugawa, F. Salces-Carcoba, A. R. Perry, Y. Yue, and I. B. Spielman, Second Chern number of a quantum-simulated nonAbelian Yang monopole, Science 360, 1429 (2018).

[57] P. Roushan, C. Neill, Y. Chen, M. Kolodrubetz, C. Quintana, N. Leung, M. Fang, R. Barends, B. Campbell, Z. Chen, B. Chiaro, A. Dunsworth, E. Jeffrey, J. Kelly, A. Megrant, J. Mutus, P. J. J. O’Malley, D. Sank, A. Vainsencher, J. Wenner, T. White, A. Polkovnikov, A. N. Cleland, and J. M. Martinis, Observation of topological transitions in interacting quantum circuits, Nature (London) 515, 241 (2014).

[58] R. Ozeri, N. Katz, J. Steinhauer, and N. Davidson, Colloquium: Bulk Bogoliubov excitations in a Bose-Einstein condensate, Rev. Mod. Phys. 77, 187 (2005). 